\title{
Porta-enxertos para a tangerineira 'Michal' no Rio Grande do Sul
}

\author{
Rootstocks for 'Michal' tangerine in Rio Grande do Sul - Brazil
}

\author{
Eduardo Cesar Brugnara ${ }^{\mathrm{I}}$ Sergio Francisco Schwarz ${ }^{\mathrm{I}}{ }^{*}$ Otto Carlos Koller II Renar João Bender ${ }^{\mathrm{II}}$ \\ Roberto Luis Weiler ${ }^{I}$ Mateus Pereira Gonzatto ${ }^{I}$ Gilmar Schäfer ${ }^{I I}$ Flávia Targa Martins ${ }^{\mathrm{I}}$ \\ Jurandir Gonçalves de Lima ${ }^{\text {III }}$
}

\section{RESUMO}

A tangerineira 'Michal' (Citrus clementina $\times$ C. tangerina) poderá ser uma boa alternativa ao Rio Grande do Sul como cultivar copa para produção de frutos em época precoce. No entanto, há falta de informações sobre seu comportamento em cultivo nas condições ambientais desse Estado. O objetivo deste trabalho foi avaliar o desenvolvimento das plantas, a produção e a qualidade dos frutos da tangerineira 'Michal' enxertada sobre cinco porta-enxertos: citrangeiro 'Troyer', citrumeleiro 'Swingle', limoeiro 'Cravo', tangerineira 'Sunki' e trifoliata 'Flying Dragon', na Depressão Central do Rio Grande do Sul. Foram avaliados os seguintes parâmetros: a altura das plantas, a circunferência do tronco, a área de projeção da copa (APC), o número e a massa (MF) de frutos produzidos, a relação MF/APC (IP), o teor de sólidos solúveis totais (SST), a acidez total titulável (ATT) e a relação SST/ATT do suco, além do tamanho dos frutos e do rendimento de suco. O citrumeleiro 'Swingle' promoveu bom vigor, boa produção, bom IP, bons níveis de SST e de ATT da 'Michal', enquanto o limoeiro 'Cravo' conferiu bom vigor, boa produção, bom IP e bom tamanho dos frutos, ambos podendo ser indicados como porta-enxertos em pomares de tangerineira 'Michal'.

Palavras-chave: Citrus, produtividade, qualidade.

\section{ABSTRACT}

'Michal' tangerine (Citrus clementina $\times$ C. tangerina) can be a good choice as canopy for early maturing fruit production in Rio Grande do Sul. The limitation is the lack of information about its performance and management. The aim of this research was to evaluate the development, production and fruit quality of 'Michal' tangerine grafted on five rootstocks: 'Troyer' citrange, 'Swingle' citrumelo, 'Cravo' lemon, 'Sunki' tangerine and 'Flying Dragon' trifoliate orange, in the Depressão Central of Rio Grande do Sul. The following variables were evaluated: plant height; trunk circumference; canopy projection area (APC); produced fruit number and weight (MF); MF/APC relation (IP); juice total soluble solids (SST) and total titrable acidity (ATT); fruit size and juice content. 'Swingle' citrumelo provided good vigor, yield, IP, SST and ATT for 'Michal', while 'Cravo' lemon induced good vigor, yield, IP and fruit size. Both are good recommendations as rootstock for orchards of 'Michal'.

Key words: Citrus, yield efficiency, fruit quality.

\section{INTRODUÇÃO}

A produção mundial de frutas cítricas foi de 108 milhões de toneladas em 2006, em uma área cultivada de aproximadamente sete milhões de hectares. O Brasil produziu cerca de 21 milhões de toneladas, situando-se como o maior produtor, com $19 \%$ da produção mundial (FAO, 2008; IBGE, 2008). Das 25 milhões de toneladas de tangerinas produzidas no mundo em 2006, 1,27 milhão de toneladas foram produzidas pelo Brasil. Em 2005, o país exportou cerca de 12 mil toneladas, faturando mais de 6,2 milhões de dólares.

O Estado do Rio Grande do Sul, terceiro maior produtor de tangerinas do Brasil, apresenta

'Programa de Pós-graduação em Fitotecnia, Universidade Federal do Rio Grande do Sul (UFRGS). Porto Alegre, RS, Brasil.

"Departamento de Horticultura e Silvicultura, Programa de Pós-graduação em Fitotecnia, UFRGS. Av. Bento Gonçalves, 7712, 91540-000, Porto Alegre, RS, Brasil. E-mail: schwarz@ufrgs.br. *Autor para correspondência.

"IIPanoramas Citrus, Butiá, RS, Brasil. 
condições de clima e solo favoráveis à produção de frutas cítricas de mesa, as quais são de excelente qualidade físico-química (KOLLER, 1994). A área colhida de tangerineiras, em 2006, foi de 13.197 hectares, e a produção foi de 172.807 toneladas de frutos (IBGE, 2008). As principais variedades cultivadas no Estado, segundo a ordem de colheita, são Okitsu, Caí, Ponkan, Pareci, Montenegrina e Murcott (JOÃO \& CONTE, 2007). A cultivar 'Michal', de origem israelense, considerada um híbrido natural entre as tangerineiras 'Clementina' e 'Dancy', produz frutos grandes, de casca avermelhada, fáceis de descascar, de elevado teor de suco, o qual apresenta excelente equilíbrio acidez/ açúcares (SAUNT, 1992). Esta pode ser uma alternativa para produção comercial de frutos de boa qualidade, com período de colheita entre as tangerineiras 'Okitsu' e 'Caí'. A limitação da expansão de sua área de cultivo ainda é a carência de informações sobre seu comportamento agronômico, já que sua introdução no Estado é relativamente recente, o que se deu por meio de sementes procedentes de Israel em 1988.

A pouca diversificação de porta-enxertos associada a outros fatores colocam em risco a citricultura do Rio Grande do Sul. Desse fato surge a necessidade de seleção de materiais adaptados e com tolerância, principalmente, a doenças (SCHÄFER et al., 2001a). O porta-enxerto influencia sensivelmente o desenvolvimento da cultivar copa de citros (KOLLER, 1994). Estudos com diversas cultivares demonstram influência do porta-enxerto no teor de nutrientes das suas folhas, na produção e no desenvolvimento da copa, no tamanho e na quantidade de suco dos frutos, no teor de sólidos solúveis totais e na acidez do suco (SALDANHA et al., 1992; LEDO et al., 1999; SCHÄFER et al. 2001b; JOUBLAN \& CORDERO, 2002; SMITH et al. 2004; NÚÑEZ et al., 2007).

O objetivo deste trabalho foi avaliar o desenvolvimento da planta, a produção e a qualidade dos frutos da tangerineira 'Michal' enxertada sobre cinco porta-enxertos nas condições climáticas da Depressão Central do Rio Grande do Sul.

\section{MATERIAL E MÉTODOS}

O ensaio foi instalado em agosto de 1997, na Empresa Panoramas Citrus, Município de Butiá, Rio Grande do Sul, Brasil (latitude 30 07’ 12”'S, longitude $51^{\circ} 57^{\prime} 45^{\prime}$ ' W, altitude de $35 \mathrm{~m}$ ). O clima local, pela classificação de Koeppen, é do tipo Cfa (MORENO, 1961), com temperatura média anual entre 18 e $19^{\circ} \mathrm{C}$, temperatura média do mês mais quente (janeiro) de $24^{\circ} \mathrm{C}$ e do mais frio (julho) de $13^{\circ} \mathrm{C}$. A precipitação média anual se situa entre 1300 e 1400mm (INSTITUTO DE PESQUISAS AGRONÔMICAS, 1989). O solo é um ARGISSILO VERMELHO Distrófico (SISTEMA..., 1999), de substrato granito, em campo nativo, com relevo ondulado.

O delineamento experimental utilizado foi o de blocos casualizados, com cinco tratamentos e quatro repetições, usando quatro plantas por parcela. O espaçamento entre plantas foi de 6 x 2,5m, utilizando mudas de raiz nua, da cultivar de tangerineira 'Michal' (Citrus clementina Hort ex Tanaka x C. tangerina Hort ex Tanaka) enxertada sobre os porta-enxertos (tratamentos): citrangeiro 'Troyer' (C. sinensis Osb. $x$ Poncirus trifoliata Raf.), citrumeleiro 'Swingle' (C. paradisi Macf. x P. trifoliata Raf.), limoeiro ‘Cravo’ (C. limonia Osb.), tangerineira 'Sunki’ (C. sunki Hort. ex Tanaka) e ‘Flying Dragon’ (P. trifoliata var. monstrosa Swing).

Antes do plantio, o solo foi corrigido, subsolado e lavrado ao longo das futuras linhas de plantas. Posteriormente, os tratos culturais, envolvendo podas, adubação, controle de ervas daninhas e doenças, foram uniformes em todas as parcelas.

As variáveis de crescimento de planta analisadas foram: a circunferência do tronco (CT), medida em cm, cinco centímetros acima do ponto de enxertia, a área de projeção da copa (APC), expressa em m², e a altura da planta, medida em metros. Aárea de projeção da copa foi calculada, a partir das medidas do diâmetro da copa nos sentidos longitudinal e transversal à linha de plantio, pela equação: $\mathrm{APC}=\pi \times$ $((\mathrm{DT}+\mathrm{DL}) / 4)^{2}$, em que DT é o diâmetro da copa transversal à linha de plantio e DL é o diâmetro longitudinal à linha. APC e altura foram determinadas em fevereiro de 2004, janeiro de 2005 e janeiro de 2006 ( $7^{\circ}, 8^{\circ}$ e $9^{\circ}$ anos do pomar). Para avaliar a produção e a produtividade das plantas, foi contado o número de frutos (NF), foi medida a massa de frutos (MF) produzidos por planta e foi determinado o índice de produtividade (IP), calculado pela relação MF/APC nos anos de 2004, 2005 e 2006. O NF e a MF foram avaliados em maio de 2001, abril de 2003, maio de 2004, junho de 2005, maio de 2006 e maio de 2007.

Nos anos de 2003, 2004 e 2005, amostras de 20 frutos foram submetidas a determinações do diâmetro, da altura e da massa média dos frutos, sendo os primeiros expressos em centímetros e a última, em 
gramas. A partir de amostras de 10 frutos foram determinados o rendimento de suco (\%), a acidez total titulável (ATT), expressa em \% de ácido cítrico, o teor de sólidos solúveis totais do suco (SST), medido em ${ }^{\circ}$ Brix, e o índice de maturação (IM), obtido por meio da relação SST/ATT. AATT foi determinada por titulação de cerca de 6,0g de suco com solução de $\mathrm{NaOH} 0,1 \mathrm{~N}$ até ser atingido o pH 8,1, seguida pelo cálculo da porcentagem de ácido cítrico por meio da equação: ATT $=[(\mathrm{ml}$ de $\mathrm{NaOH}) \times($ concentração Normal do $\mathrm{NaOH}) \times 0,064 \times 100] /($ gramas de suco $)$. O SST foi determinado por refratometria.

Os dados coletados foram avaliados por análise de variância $(\mathrm{P}=0,05)$, e as médias foram comparadas pelo teste de Duncan, utilizando o aplicativo SAS ${ }^{\circledR}$.

\section{RESULTADOS E DISCUSSÃO}

Na análise de variância, a interação entre ano e tratamento foi significativa para o NF, a MF, o IP e o diâmetro, a altura e a massa média dos frutos. Não houve interação significativa entre anos e tratamentos, para a CT, APC e altura das plantas. Na média, durante três anos, de 2004 a 2006 (Tabela 1), a altura das plantas enxertadas sobre o 'Flying Dragon' foi menor do que nos demais porta-enxertos, e entre estes as diferenças não foram significativas. Quanto à CT, O limoeiro 'Cravo', o citrumeleiro 'Swingle' e a tangerineira 'Sunki' revelaram-se mais vigorosos do que o citrangeiro 'Troyer' e o ‘Flying Dragon', havendo indícios de que, com a APC, acontece o mesmo, porém as diferenças não alcançaram nível de significância estatística.

Efeitos diferentes dos porta-enxertos sobre o desenvolvimento das copas são comuns, com

Tabela 1 - Altura, área de projeção da copa (APC) e circunferência do tronco (CT) de tangerineiras 'Michal' enxertadas sobre diferentes portaenxertos - média de três anos (2004 a 2006). Butiá, RS.

\begin{tabular}{lccc}
\hline Porta-enxertos & Altura (m) & APC $\left(\mathrm{m}^{2}\right)$ & CT $(\mathrm{cm})$ \\
\hline 'Cravo' & $2,14 \mathrm{a}$ & 3,25 & $30,39 \mathrm{a}$ \\
'Swingle' & $2,12 \mathrm{a}$ & 3,25 & $29,29 \mathrm{ab}$ \\
'Troyer' & $1,96 \mathrm{a}$ & 1,89 & $22,32 \mathrm{bc}$ \\
'Sunki' & $1,91 \mathrm{a}$ & 2,20 & $26,24 \mathrm{ab}$ \\
'Flying Dragon' & $1,56 \mathrm{~b}$ & 1,46 & $17,11 \mathrm{c}$ \\
CV(\%) & 6,80 & 30,27 & 15,23 \\
\hline
\end{tabular}

Médias não seguidas pela mesma letra na coluna não diferem pelo teste de Duncan, a 5\% de probabilidade de erro. cultivares 'copa’ e em ambientes diferentes. O portaenxerto ‘Flying Dragon’ é conhecido como ananizante, e o menor vigor das plantas de 'Michal' enxertadas sobre ele já foi observado por NUNES et al. (2002). MARTINS (2005) verificou que o porta-enxerto $\boldsymbol{P}$. trifoliata induziu menor volume de copa à tangerineira 'Montenegrina' do que o citrumeleiro 'Swingle' e o citrangeiro ‘Troyer’. PARENTE et al. (1993), estudando a influência de porta-enxertos, verificou que a tangerineira ‘Ponkan' (C. reticulada Blanco) foi menos vigorosa sobre $\boldsymbol{P}$. trifoliata, enquanto que sobre citrangeiro 'Troyer' apresentou médio vigor, o que confere com os resultados deste trabalho.

Quanto à produção (NF e MF), nos anos de 2004 e 2005, os tratamentos 'Swingle' e 'Cravo' proporcionaram as maiores produções (Tabela 2). Nos demais anos, as diferenças entre porta-enxertos não atingiram nível de significância, provavelmente pelo alto coeficiente de variação, principalmente em 2001 e 2003, quando as plantas eram bem novas.

Quanto ao IP (Tabela 3), com pequenas variações nos três anos avaliados, pela ordem, o 'Swingle’ e o ‘Cravo' apresentaram os índices mais elevados de produtividade da copa 'Michal', enquanto 'Sunki' conferiu os menores índices. NUNES et al. (2002) não observaram diferenças em razão dos portaenxertos no índice de produtividade de tangerineiras 'Michal'. FIGUEIREDO et al. (1997) observou alto coeficiente de variação dos dados coletados, não tendo verificado diferenças significativas para a variável "produção por metro cúbico de copa” da variedade copa Murcott.

As variáveis altura, diâmetro e massa média dos frutos (Tabela 4) apresentaram diferença significativa, em 2005, e diâmetro também em 2004. Em geral, no ano de 2005, os frutos de maior tamanho e massa média foram os frutos produzidos nas plantas sobre 'Cravo' e 'Troyer'. Em regra, o aumento do número de frutos produzidos causa redução em seu tamanho e sua massa média, por causa do limitado fornecimento de fotoassimilados a cada fruto. Isso poderia explicar o menor tamanho e a menor massa média dos frutos produzidos pelo 'Swingle', que, como pode ser observado na tabela 2, em 2005, proporcionou produção superior aos demais porta-enxertos. Porém, não houve correlação significativa entre NF e massa média dos frutos em 2005, o que sugere que as diferenças, naquele ano, também devem ter tido outra causa. A adaptação ao ambiente pode explicar esse fenômeno, pois diferentes genótipos respondem 
Tabela 2 - Número e massa de frutos produzidos por tangerineiras 'Michal' enxertadas em diferentes porta-enxertos, no $4^{\mathrm{o}}$ ano (2001) e do $6^{0}$ a $10^{\circ}$ ano (2003 a 2007) de idade do pomar. Butiá, RS.

\begin{tabular}{|c|c|c|c|c|c|c|}
\hline \multirow{2}{*}{ Porta-enxerto } & & \multicolumn{4}{|c|}{---------Ano------------------------------- } & \\
\hline & 2001 & 2003 & 2004 & 2005 & 2006 & 2007 \\
\hline & \multicolumn{6}{|c|}{------------------------------------------------Número de frutos por planta--------------------------------------------- } \\
\hline ‘Cravo’ & 74,5 & 190,2 & 296,4 a & 101,4 bc & 37,4 & 56,7 \\
\hline 'Flying Dragon’ & 9,6 & 59,0 & $100,3 \mathrm{ab}$ & $47,6 \mathrm{c}$ & 13,1 & 43,6 \\
\hline 'Sunki' & 0,0 & 0,2 & $79,1 \mathrm{~b}$ & $133,8 \mathrm{~b}$ & 40,8 & 31,4 \\
\hline 'Swingle' & 25,1 & 118,2 & $270,4 \mathrm{a}$ & $349,1 \mathrm{a}$ & 73,2 & 12,6 \\
\hline ‘Troyer' & 4,6 & 25,5 & $114,1 \mathrm{ab}$ & 79,5 bc & 34,7 & 44,9 \\
\hline \multirow[t]{2}{*}{ CV (\%) } & 83,30 & 76,80 & 29,11 & 18,26 & 35,00 & 19,20 \\
\hline & \multicolumn{6}{|c|}{------------------------------------------Massa de frutos por planta (kg) ------------------------------------------ } \\
\hline ‘Cravo’ & 10,5 & 25,7 & 36,8 a & $16,97 \mathrm{~b}$ & 5,97 & 9,05 \\
\hline 'Flying Dragon’ & 1,6 & 8,5 & 12,8 bc & $6,02 \mathrm{~b}$ & 2,11 & 0,81 \\
\hline 'Sunki' & 0,0 & 0,0 & $11,6 \mathrm{c}$ & $16,83 \mathrm{~b}$ & 3,32 & 4,07 \\
\hline 'Swingle' & 3,4 & 15,8 & $33,6 \mathrm{ab}$ & $40,01 \mathrm{a}$ & 13,78 & 2,73 \\
\hline ‘Troyer’ & 0,6 & 3,5 & 13,4 bc & $11,08 \mathrm{~b}$ & 11,58 & 6,65 \\
\hline CV(\%) & 53,93 & 60,46 & 25,62 & 18,77 & 31,26 & 17,66 \\
\hline
\end{tabular}

Médias não seguidas pela mesma letra na coluna diferem pelo teste de Duncan, a 5\% de probabilidade de erro.

distintamente aos estresses e estímulos ambientais, como a deficiência hídrica, à qual o limoeiro ‘Cravo’ é considerado tolerante, tanto que NUNES et al. (2002), testando os mesmos porta-enxertos usados neste trabalho, verificaram que a tangerineira 'Michal' enxertada sobre limoeiro 'Cravo' produziu frutos maiores do que os de plantas enxertadas sobre os outros porta-enxertos. A massa média dos frutos, acima de $120 \mathrm{~g}$, assegura que a copa 'Michal' produz frutos de tamanho adequado ao mercado de fruta fresca, nas condições avaliadas.

Na média dos anos avaliados, a ATT e o SST, no tratamento 'Cravo', foram menores que nos demais tratamentos, exceto 'Sunki' (Tabela 5). Não houve diferenças significativas para a relação SST/

Tabela 3 - Índice de produtividade (IP) de tangerineiras 'Michal' enxertadas em diferentes porta-enxertos do $7^{\circ}$ ao $9^{\circ}$ ano (2004 a 2006) do pomar. Butiá, RS.

\begin{tabular}{llll}
\hline Porta-enxertos & 2004 & 2005 & 2006 \\
\hline 'Cravo' & $3,67 \mathrm{a}$ & $2,68 \mathrm{~b}$ & $1,99 \mathrm{a}$ \\
'Swingle' & $3,30 \mathrm{ab}$ & $3,84 \mathrm{a}$ & $1,62 \mathrm{a}$ \\
'Flying Dragon' & $3,09 \mathrm{abc}$ & $2,43 \mathrm{~b}$ & $1,96 \mathrm{a}$ \\
'Troyer' & $2,76 \mathrm{bc}$ & $2,90 \mathrm{~b}$ & $2,16 \mathrm{a}$ \\
'Sunki' & $2,55 \mathrm{c}$ & $3,01 \mathrm{~b}$ & $1,32 \mathrm{~b}$ \\
CV(\%) & 11,84 & 13,73 & 18,30 \\
\hline
\end{tabular}

Médias não seguidas pela mesma letra na coluna diferem pelo teste de Duncan, a 5\% de probabilidade de erro.
ATT (IM). Esses dados indicam que os frutos dos tratamentos 'Flying Dragon', 'Troyer' e 'Swingle' apresentaram melhor qualidade, ao passo que o teor de suco dos frutos não foi influenciado pelos portaenxertos e foi suficientemente alto para atingir bom padrão de comercialização (>40\%) (Tabela 5).

A influência de porta-enxertos sobre índices qualitativos dos frutos produzidos pelas variedades copa também foi verificada em outras pesquisas. No Chipre, tangerineiras 'Clementina' enxertadas sobre citrumeleiro 'Swingle' e citrangeiro 'Troyer' produziram frutos cujo teor de sólidos solúveis totais superou grande parte dos outros porta-enxertos (GEORGIOU, 2002). SMITH et al. (2004), na Austrália, observaram efeito dos porta-enxertos no rendimento de suco, teor de sólidos solúveis totais do suco, peso e diâmetro dos frutos da tangerineira 'Ellendale', onde o citrangeiro 'Troyer' conferiu maior teor de sólidos solúveis totais do que o limoeiro 'Rugoso', a laranjeira doce e a tangerineira 'Emperor'.

Verificou-se, no Rio Grande do Sul, que os frutos da tangerineira 'Michal' atingem padrões para comercialização como fruta para consumo in natura; com rendimento de suco superior a $40 \%$ e relação SST/ ATT superior a 8,0.

\section{CONCLUSÕES}

Os porta-enxertos limoeiro 'Cravo', citrumeleiro ‘Swingle’ e tangerineira 'Sunki’ conferem 
Tabela 4 - Tamanho e massa média dos frutos de tangerineiras 'Michal' enxertadas em cinco porta-enxertos (2003 a 2005). Butiá, RS.

\begin{tabular}{lccc}
\hline Porta-enxertos & 2003 & 2004 & 2005 \\
\hline -----------------------Altura (mm) -------------------- \\
'Cravo' & 55,64 & 51,7 & $58,77 \mathrm{a}$ \\
'Troyer' & 55,32 & 51,99 & $57,27 \mathrm{a}$ \\
'Flying Dragon' & 55,22 & 53,63 & $53,85 \mathrm{~b}$ \\
'Sunki' & - & 54,31 & $53,65 \mathrm{~b}$ \\
'Swingle' & 54,93 & 54,99 & $52,89 \mathrm{~b}$ \\
CV (\%) & 1,99 & 3,03 & 1,97 \\
------------------------Diâmetro (mm) ------------------------- \\
'Cravo' & 68,77 & $66,52 \mathrm{a}$ & $72,95 \mathrm{a}$ \\
'Troyer' & 69,34 & $64,45 \mathrm{~b}$ & $71,31 \mathrm{a}$ \\
'Flying Dragon' & 70,01 & $66,56 \mathrm{a}$ & $67,92 \mathrm{a}$ \\
'Sunki' & - & $67,39 \mathrm{a}$ & $67,22 \mathrm{~b}$ \\
'Swingle' & 67,74 & $66,07 \mathrm{ab}$ & $65,96 \mathrm{~b}$ \\
CV (\%) & 1,97 & 1,62 & 2,43 \\
----------------------------Massa (g) ---------------------------- \\
'Cravo' & 145,56 & 126,67 & $169,16 \mathrm{a}$ \\
'Troyer' & 148,21 & 122,33 & $160,16 \mathrm{a}$ \\
'Flying Dragon' & 148,96 & 131,86 & $139,92 \mathrm{~b}$ \\
'Sunki’ & - & 137,29 & $135,22 \mathrm{~b}$ \\
'Swingle' & 141,12 & 132,89 & $128,07 \mathrm{~b}$ \\
CV (\%) & 4,72 & 4,73 & 5,98 \\
\hline
\end{tabular}

Médias não seguidas pela mesma letra na coluna diferem pelo teste de Duncan, a 5\% de probabilidade de erro.

maior vigor à copa da tangerineira 'Michal'. Já o limoeiro 'Cravo' e o citrumeleiro 'Swingle' conferem maior produção por planta na fase jovem do pomar, além de induzirem as melhores combinações das características vigor, produtividade e qualidade de frutos, podendo ser indicados para uso comercial como porta-enxerto dessa cultivar copa.

Tabela 5 - Acidez total titulável (ATT), teor de sólidos solúveis totais (SST), índice de maturação (IM) e porcentagem de suco dos frutos de tangerineiras 'Michal' enxertadas em cinco porta-enxertos - média de quatro anos (2003 a 2006). Butiá, RS.

\begin{tabular}{lcccc}
\hline Tratamento & ATT & SST $\left({ }^{\circ}\right.$ Brix $)$ & IM & $\%$ Suco \\
\hline 'Cravo' & $0,73 \mathrm{~b}$ & $8,98 \mathrm{~b}$ & 12,25 & 50,22 \\
'Flying Dragon' & $0,86 \mathrm{a}$ & $10,12 \mathrm{a}$ & 12,02 & 50,27 \\
'Sunki' & $0,80 \mathrm{ab}$ & $9,64 \mathrm{a}$ & 11,85 & 49,19 \\
'Swingle' & $0,85 \mathrm{a}$ & $9,94 \mathrm{a}$ & 11,80 & 50,90 \\
'Troyer' & $0,85 \mathrm{a}$ & $9,74 \mathrm{a}$ & 11,62 & 50,62 \\
CV (\%) & 5,67 & 2,71 & 6,41 & 5,32 \\
\hline
\end{tabular}

Médias não seguidas pela mesma letra na coluna diferem pelo teste de Duncan, a 5\% de probabilidade de erro.

\section{AGRADECIMENTO}

Ao Conselho Nacional de Desenvolvimento Científico e Tecnológico (CNPq) e à Coordenação de Aperfeiçoamento de Pessoal de Nível Superior (CAPES), pelo apoio financeiro.

\section{REFERÊNCIAS}

FAO. Agricultural Data - FAOSTAT. Disponível em: http:/ /faostat.fao.org/site/291/default.aspx. On line. Acesso em: 21 jul. 2008.

FIGUEIREDO, J.O. et al. Produções iniciais de Tangor 'Murcott' sobre 16 porta-enxertos, na região de Itirapina, SP. Revista Brasileira de Fruticultura, Cruz das Almas, v.19, n.2, p.191-197, 1997.

GEORGIOU, A. Evaluation of rootstocks for 'Clementine' mandarin in Cyprus. Scientia Horticulturae, Amsterdan, v.93, n.1, p.29-38, 2002.

IBGE. Tabela 1613 - Quantidade produzida, Valor da produção, Área plantada e Área colhida da lavoura permanente. Disponível em: http://www.sidra.ibge.gov.br/bda/ tabela/listabl.asp?c=1613\&z=t\&o=11. On line. Acesso em: 21 jul. 2008.

INSTITUTO DE PESQUISAS AGRONÔMICAS. Seção de Ecologia Agrícola. Atlas agroclimático do Estado do Rio Grande do Sul. Porto Alegre, 1989. 3v.il.

JOUBLAN, J.P.; CORDERO, N. Comportamiento de algunos cítricos sobre diferentes portainjertos, en su tercera temporada de crecimiento, Quillón VIII Región, Chile. Agricultura Técnica, Santiago do Chile, v.62, n.3, p.469-479, 2002.

KOLLER, O.C. Citricultura: laranja, limão e tangerina. Porto Alegre: Rigel, 1994. 446p.

JOÃO, P.L.; CONTE, A. Levantamento da fruticultura comercial do Rio Grande do Sul: 2006. Porto Alegre: EMATER/RS-ASCAR, 2007. 83p.

LEDO, A.S. et al. Porta-enxertos para laranjeiras-doces (Citrus sinensis (L.) Osb.), em Rio Branco, Acre. Pesquisa Agropecuária Brasileira, Brasília, v.34, n.7, p.1211-1216, 1999.

MARTINS, F.T. Comportamento de laranjeira 'Valência' e tangerineira 'Montenegrina' propagadas por estaquia e enxertia. 2005. 67f. Dissertação (Mestrado em Fitotecnia) - Faculdade de Agronomia, Universidade Federal do Rio Grande do Sul.

MORENO, J.A. Clima do Rio Grande do Sul. Porto Alegre: Secretaria de Agricultura, 1961. 38p.

NUNES, D.S. et al. Produção e desenvolvimento vegetativo de plantas jovens de tangerineira 'Michal' enxertadas sobre sete porta-enxertos. In: CONGRESSO BRASILEIRO DE FRUTICUlTURA, 17., 2002, Belém, PA. Anais... Belém : SBF, 2002. 1 CD. 
NÚÑEZ, E.E. et al. Desenvolvimento vegetativo, produção e qualidade de frutos da tangerina 'Fremont' sobre quatro portaenxertos. Revista Brasileira de Fruticultura, Jaboticabal, v.29, n.2, p.308-312, 2007. Disponível em: http:// www.scielo.br/scielo.php?script=sci_arttext\&pid=S010029452007000200023\&lng=pt\&nrm=iso. Doi: $10.1590 /$ S0100-29452007000200023.

PARENTE, T.V. et al. Comportamento da tangerineira Ponkan (Citrus reticulata Blanco) sobre 14 porta-enxertos no Distrito Federal. Revista Brasileira de Fruticultura, Cruz das Almas, v.15, n.1, p.35-41, 1993.

SALDANHA, E.L. et al. Comportamento do tangor 'Murcote' em 12 porta enxertos no Rio Grande do Sul. Revista Brasileira de Fruticultura, Cruz das Almas, v.14, n.3, p.105-112, 1992.

SAUNT, J. Variedades de cítricos del mundo. Valencia, Espanha: Sinclair International, 1992, 128p.il.

SCHÄFER, G. et al. Porta-enxertos utilizados na citricultura. Ciência Rural, Santa Maria, v.31, n.4, p.723-733, 2001a. Disponível em: http://www.scielo.br/scielo.php?script=sci_arttext\&pid=S0103- 84782001000400028\&lng=pt\&nrm=iso\&tlng=pt. Doi: $10.1590 /$ S0103-84782001000400028.

SCHÄFER, G. et al. Produção e desenvolvimento da tangerineira 'Montenegrina' propagada por enxertia e estaquia, no Rio Grande do Sul. Revista Brasileira de Fruticultura, Jaboticabal, v.23, n.3, p. 668-672, 2001b. Disponível em: http://www.scielo.br/scielo.php?script=sci_arttext\&pid=S0100 29452001000300045\&lng=pt\&nrm=iso\&tlng=pt. Doi: 10.1590/S0100-29452001000300045.

SISTEMA Brasileiro de Classificação de Solos. Brasília: EMBRAPA-SPI, 1999. 412p.

SMITH, M.W. et al. Long-term performance of 'Ellendale' mandarin on seven commercial rootstocks in sub-tropical Australia. Scientia Horticulturae, Amsterdan, v.102, n.1, p.75-89, 2004. Disponível em: http://www.sciencedirect.com/ science?_ob=MImg\&_imagekey=B6TC3-4C0071D-3F \&_cd i $=5159 \&$ \&_us e r $=687304 \&$ \&_orig $=$ b r o w

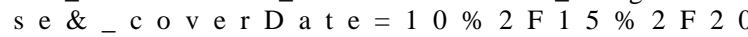
$04 \&$ s k $=998979998 \&$ vie w $=$ c \& w chp $=$ d G L b V l W zSkzS\&md5=97f21a9a98a7083d047899d12db7e651\&ie=/ sdarticle.pdf. Doi: 10.1016/j.scienta.2003.12.004. 